\title{
Decoding music-evoked emotions in the auditory and motor cortex
}

\section{Putkinen, $\mathrm{V}$}

http://hdl.handle.net/10026.1/17629

\section{$10.1101 / 2020.05 .24 .101667$}

All content in PEARL is protected by copyright law. Author manuscripts are made available in accordance with publisher policies. Please cite only the published version using the details provided on the item record or document. In the absence of an open licence (e.g. Creative Commons), permissions for further reuse of content should be sought from the publisher or author. 


\title{
Decoding Music-Evoked Emotions in the Auditory and Motor Cortex
}

\author{
Vesa Putkinen ${ }^{1}{ }^{1}$, Sanaz Nazari-Farsani ${ }^{1}$, Kerttu Seppälä ${ }^{1}$, \\ Tomi Karjalainen ${ }^{1}{ }^{1}$, Lihua Sun ${ }^{1}$, Henry K. Karlsson ${ }^{1}$, Matthew Hudson ${ }^{1,4}$, \\ Timo T. Heikkilä ${ }^{3}$, Jussi Hirvonen ${ }^{1,2}$ and Lauri Nummenmaa ${ }^{1,3}$
}

${ }^{1}$ Turku PET Centre, and Turku University Hospital, University of Turku, 20520, Turku, Finland, ${ }^{2}$ Department of Radiology, Turku University Hospital, 20520, Turku, Finland, ${ }^{3}$ Department of Psychology, University of Turku, FI-20014, Turku, Finland and ${ }^{4}$ National College of Ireland, D01 K6W2, Dublin, Ireland

Address correspondence to Vesa Putkinen, Turku PET Centre, and Turku University Hospital, University of Turku, Finland. Email: vesa.putkinen@utu.fi.

\begin{abstract}
Music can induce strong subjective experience of emotions, but it is debated whether these responses engage the same neural circuits as emotions elicited by biologically significant events. We examined the functional neural basis of music-induced emotions in a large sample $(n=102)$ of subjects who listened to emotionally engaging (happy, sad, fearful, and tender) pieces of instrumental music while their hemodynamic brain activity was measured with functional magnetic resonance imaging (fMRI). Ratings of the four categorical emotions and liking were used to predict hemodynamic responses in general linear model (GLM) analysis of the fMRI data. Multivariate pattern analysis (MVPA) was used to reveal discrete neural signatures of the four categories of music-induced emotions. To map neural circuits governing non-musical emotions, the subjects were scanned while viewing short emotionally evocative film clips. The GLM revealed that most emotions were associated with activity in the auditory, somatosensory, and motor cortices, cingulate gyrus, insula, and precuneus. Fear and liking also engaged the amygdala. In contrast, the film clips strongly activated limbic and cortical regions implicated in emotional processing. MVPA revealed that activity in the auditory cortex and primary motor cortices reliably discriminated the emotion categories. Our results indicate that different music-induced basic emotions have distinct representations in regions supporting auditory processing, motor control, and interoception but do not strongly rely on limbic and medial prefrontal regions critical for emotions with survival value.
\end{abstract}

Key words: emotion, fMRI, music, MVPA, pattern classification

\section{Introduction}

Human attraction to music stems from the aesthetic experience and strong emotional reactions music evokes. Basic emotion theories posit a finite number of innate and universal emotions that have evolved because they promote survival by motivating adaptive behaviors and help maintaining physiological homeostasis (Ekman 1992), and numerous neuroimaging studies have tested whether music is capable of activating the survival circuits underlying these emotions (Mitterschiffthaler et al. 2007; Brattico et al. 2011; Koelsch et al. 2013). Indeed, emotional responses to music show many hallmarks of basic emotions including distinct subjective experience, emotional expression, action tendencies, changes in the autonomic nervous system activation (Juslin and Vastfjall 2008) and distinct effects of thought and judgment (Vuoskoski and Eerola 2012). Yet, it seems counterintuitive that that instrumental music could elicit "genuine" basic emotions because emotional reactions to music do not have unambiguous causes (real-life threat, success, or loss, etc.) and they do not involve events that have obvious goal or survival-relevant consequences (Konečni 2008; Scherer and Zentner 2008). Thus, even though listeners often interpret their 
subjective reactions to music as basic emotions, these responses may differ from genuine basic emotions in the underlying neural processes.

Some acoustic features contributing to different musicinduced emotions may be universally recognizable (Fritz et al. 2009), perhaps because similar cues communicate emotion in human vocalizations and music (Juslin and Laukka 2003; Frühholz et al. 2016). These features may recruit partly the same neural networks across the domains (Peretz 2010; Escoffier et al. 2013). Music-induced emotions are nevertheless dependent on cultural learning (Higgins 2012; McDermott et al. 2016) and therefore may engage partly distinct neural systems than the putatively innate basic emotions.

Emotions elicited by biologically salient stimuli consistently engage subcortical structures such as the amygdala, thalamus, striatum, and brain stem and cortical regions such as the anterior cingulate, insula, and medial and orbital frontal cortices (Kober et al. 2008; De La Vega et al. 2016). A recent meta-analysis indicates that different music-induced emotions also activate these structures suggesting that these emotions may rely on some of the same evolutionarily adaptive mechanisms that underlie emotions with survival value (Koelsch 2014). However, it is unclear whether the activation of these circuits discriminates between specific categories of music-induced emotions. Thus far, fMRI studies have largely failed to find reliable categoryspecific correlates of music-induced emotions and often contradict each other in terms of the regions they implicate in specific music-induced emotions such as happiness (Mitterschiffthaler et al. 2007; Brattico et al. 2011), sadness (Mitterschiffthaler et al. 2007; Trost et al. 2012), or fear (Koelsch et al. 2013; Bogert et al. 2016).

The mass univariate general linear model (GLM) has been the dominating analysis approach in fMRI studies on emotions. More recently, multivariate pattern analysis (MVPA) has been adopted in order to decode high-dimensional regularities in fMRI data that predict different basic and social emotions (Nummenmaa and Saarimäki 2017). These studies indicate that discrete emotions perceived in facial expressions, bodily movements, and vocalizations (Ethofer et al. 2009; Peelen et al. 2010; Volynets et al. 2020) can be decoded from brain activity in sensory cortices while emotional states induced by stories, movies, and imagery are represented in discrete and widely distributed activity patterns across various cortical and subcortical regions (Sitaram et al. 2011; Kassam et al. 2013; Saarimäki et al. 2015). Thus, different music-evoked emotions might also be based on distinct but spatially overlapping neural activation patterns not captured by univariate analysis. Two prior MVPA-fMRI studies indicate that activity patterns in the auditory cortices predict whether subjects heard short bursts of violin and clarinet sounds conveying either sadness, happiness, or fear (Paquette et al. 2018; Sachs et al. 2018). These activity patterns probably reflect sensory processing of acoustic properties with different emotional connotations, but it is unclear whether such auditory cortical patterns predict different emotions actually felt by the listeners during naturalistic music-listening. A few other emotion-related MVPA-fMRI studies have used music as stimuli but either collapsed data obtained with music and other means of emotion-induction precluding inferences specifically about music-induced emotions (Kragel and LaBar 2015) or focused only on emotional valence instead of discrete music-induced emotions (Kim et al. 2017). Thus, it remains unclear whether distinct musicinduced emotions are represented in anatomically distributed activity patterns similarly to emotions elicited by non-musical stimuli.

\section{The Current Study}

Here we combined GLM and multivariate pattern analyses of fMRI data from a large sample of 102 subjects to test whether 1) different music-induced emotions reliably activate brain's emotion circuit similarly as emotions elicited by biologically salient episodes shown in videos and 2) whether music-evoked emotional responses are organized in a discrete fashion in the brain. We focused on emotions happiness, sadness, fear, and tenderness because they cover the four quadrants of the valence-arousal-circumplex, are included in categorical models frequently used to describe music-induced emotions in the literature (Zentner et al. 2008; Eerola and Vuoskoski 2010), and are rated consistently across listeners (Juslin 2013). Subjects underwent fMRI while they listened to excerpts of instrumental movie soundtracks validated previously to reliably elicit these emotions. To map neural circuits governing nonmusical emotions, we also scanned the same subjects in a previously validated emotion "localizer" paradigm (Lahnakoski et al. 2012; Karjalainen et al. 2017; Karjalainen et al. 2019) in which subjects view film clips with positive and negative emotional content. Like music, fictitious films are devoid of real-life consequences and yet elicit strong emotions. However, unlike instrumental music, movies offer a rich, multimodal simulation of the real-life emotional events. Thereby, movies provide a naturalistic paradigm for mapping neural circuits supporting emotions in real-world situations (Sonkusare et al. 2019). We found that music-induced basic emotions engaged regions supporting auditory processing, motor control, somatosensation, and interoception but did not strongly activate limbic and medial prefrontal regions that govern nonmusical emotions. Furthermore, MVPA revealed that activity in the auditory and primary motor cortices, but not in the core emotion circuits, reliably discriminated the emotion categories.

\section{Materials and Methods}

\section{Subjects}

Altogether 102 volunteers participated in the study (51 females, mean age 31 years, range 20-57 years). Fifty percent of subjects had received no formal training on a musical instrument (The median for the months of training was 6.3.) The exclusion criteria included a history of neurological or psychiatric disorders, alcohol or substance abuse, current use of medication affecting the central nervous system, and the standard MRI exclusion criteria. Two additional subjects were scanned but excluded from further analyses because unusable MRI data due to gradient coil malfunction. All subjects gave an informed, written consent and were compensated for their participation. The ethics board of the Hospital District of Southwest Finland had approved the protocol, and the study was conducted in accordance with the Declaration of Helsinki.

\section{Study Design}

The stimuli were 18 45-s (including a 10-ms fadeout) excerpts of instrumental music. Sixteen excerpts were chosen from a set of movie soundtracks based on high ratings for happiness, 
sadness, fear, or tenderness in a previous study (Eerola and Vuoskoski 2010). For each of the four emotions, four stimuli were selected that had received high rating for the target emotion and low ratings for the other three (see the Supplementary Material for details). Two additional excerpts of instrumental rock (Far Beyond the Sun by Yngwie J. Malmsteen) were included to add variation in the musical material in terms of genre and instrumentation. Instrumental music was used to minimize the contribution of semantic knowledge on the emotional responses.

A 45-s random tone sequence followed by $45 \mathrm{~s}$ of silence was presented at the beginning of the run. After this, the musical excerpts were presented in a fixed pseudo-random order without silent breaks in between. The last excerpt was followed by $45 \mathrm{~s}$ of silence, another 45 -s random tone sequence, and a third 45-s silent period (see the Supplementary Material for the contrasts between music vs. control stimuli and music vs. silence). Subjects were asked to remain still during the fMRI and focus on the feelings evoked by the music. Stimuli were presented binaurally via MRI-compatible headphones (Sensimetrics S14) at a comfortable level adjusted individually for each participant. After the scanning session, the subjects listened to each music excerpt again and rated for experience of fear, happiness, sadness, tenderness, and liking on a scale ranging from 1 to 10 (1=extremely weak, $10=$ extremely strong). Ratings were done using an online rating tool developed in-house (https://gitlab.utu.fi/tithei/petrating, Heikkilä et al. 2020). Complete ratings were obtained from 91 participants. Ratings averaged across these subjects were used as regressors in the analysis of the BOLD data (cf. Trost et al. 2012).

To map brain regions governing non-musical emotions, we used a previously validated emotion "localizer" paradigm in which the subjects viewed a compilation of 96 movie clips extracted from mainstream English language feature films (mean duration $12.5 \mathrm{~s}$; total duration $20 \mathrm{~min}$ ) containing variable emotional and non-emotional content (for details, see Karjalainen et al. 2017, 2019; Lahnakoski et al. 2012). The movie clips were presented in fixed order without breaks in between. The film clips contained scenes with displays of positive emotions (e.g., laughter, friendly discussion, expression of affection between parents and children or between romantic partners), negative emotions (e.g., crying, arguing, violence), scenes without emotional content (e.g., humans in a neutral emotional state, objects, landscapes). Dynamic ratings with a 4s temporal resolution were obtained for the intensity of positive and negative emotions observed in the film clips from a separate sample of subjects $(n=6)$ who did not participate in the fMRI study. The average ratings were subsequently used as regressors in GLM analysis. The film clips were presented via NordicNeuroLab VisualSystem binocular display (audio was delivered as described above).

\section{MRI Data Acquisition}

The MRI data were acquired using a Phillips Ingenuity TF PET/MR 3-T whole-body scanner. High-resolution $\left(1 \mathrm{~mm}^{3}\right)$ structural images were obtained with a T1-weighted sequence (TR $9.8 \mathrm{ms,}$ TE $4.6 \mathrm{~ms}$, flip angle $7^{\circ}, 250 \mathrm{~mm}$ FOV, $256 \times 256$ reconstruction matrix). A total of 407 and 470 functional volumes were acquired for the music and movie experiments, respectively, with a T2*-weighted echo-planar imaging sequence (TR $2600 \mathrm{~ms}$, TE $30 \mathrm{~ms}, 75^{\circ}$ flip angle, $240 \mathrm{~mm}$ FOV, $80 \times 80$ reconstruction matrix, $62.5 \mathrm{kHz}$ bandwidth, $3.0 \mathrm{~mm}$ slice thickness, 45 interleaved slices acquired in ascending order without gaps).

\section{Structural and Functional MRI Data Preprocessing}

MRI data were preprocessed using fMRIPprep 1.3.0.2 (Esteban et al. 2019). The following preprocessing was performed on the anatomical T1-weighted (T1w) reference image: correction for intensity non-uniformity, skull-stripping, brain surface reconstruction, and spatial normalization to the ICBM 152 Nonlinear Asymmetrical template version 2009c (Fonov et al. 2009) using nonlinear registration with antsRegistration (ANTs 2.2.0) and brain tissue segmentation. The following preprocessing was performed on the functional data: coregistration to the $\mathrm{T} 1 \mathrm{w}$ reference, slice-time correction, spatial smoothing with a 6-mm Gaussian kernel, automatic removal of motion artifacts using ICA-AROMA (Pruim et al. 2015), and resampling of the MNI152NLin2009cAsym standard space. Lowfrequency drifts were removed with a 240-s-Savitzky-Golay filter (Çukur et al. 2013).

\section{Full-Volume GLM Data Analysis}

The fMRI data analyzed in SPM12 (Wellcome Trust Center for Imaging, London, UK, http://www.fil.ion.ucl.ac.uk/spm). To reveal regions activated by music vs. silence, a general linear model (GLM) was fitted to the data where the music sequence and the silent periods were modeled with separate boxcar regressors. The regional effects of the five emotion dimensions were assessed with a GLM where the design matrix included the music boxcar regressor and each of the emotion rating regressors as parametric modulators. For the movie experiment, the average dynamic rating for the intensity of positive and negative emotions was used as a regressor in the GLM analysis. Contrast images for the main effects of music, silence, and each emotion regressor were generated for each participant and subjected to a second-level analysis. Clusters surviving family-wise error (FWE) correction $(P<0.05)$ are reported.

\section{Region-of-Interest Analyses}

Beta weights for each of the five emotion regressors were extracted from 15 anatomical regions of interest (ROI) implicated in emotional processing (Paquette et al. 2018; Saarimäki et al. 2015, 2018). These included the amygdala, caudate, putamen, pallidum, thalamus, hippocampus, insula, anterior and posterior cingulate, SMA, precentral and postcentral gyri, precuneus, frontal pole, and auditory cortex. The mean beta weights for each ROI were calculated from the first-level contrast images of each subject using ROI masks derived from the AAL atlas. The beta weights for each emotion in each ROI were compared against zero with separate one-sample $t$ tests.

\section{Multivariate Pattern Analysis}

A between-subject classification of the four emotion categories (fear, happiness, sadness, and tenderness) was performed in Python using the PyMVPA toolbox (Hanke et al. 2009). A support vector machine with radial basis function (RBF) kernel was trained to recognize the emotion categories using leave-onesubject-out cross-validation, where the classifier is trained on the data from all except one subject and tested on the holdout subject data; this procedure is repeated $\mathrm{N}$ times so that each subject is used once as the hold-out subject; this kind of 
(a)

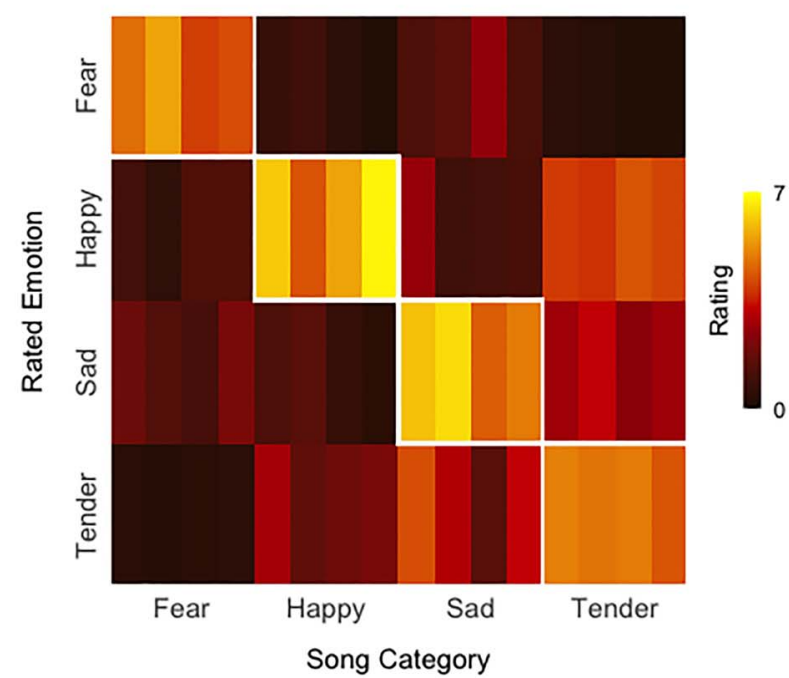

(b)

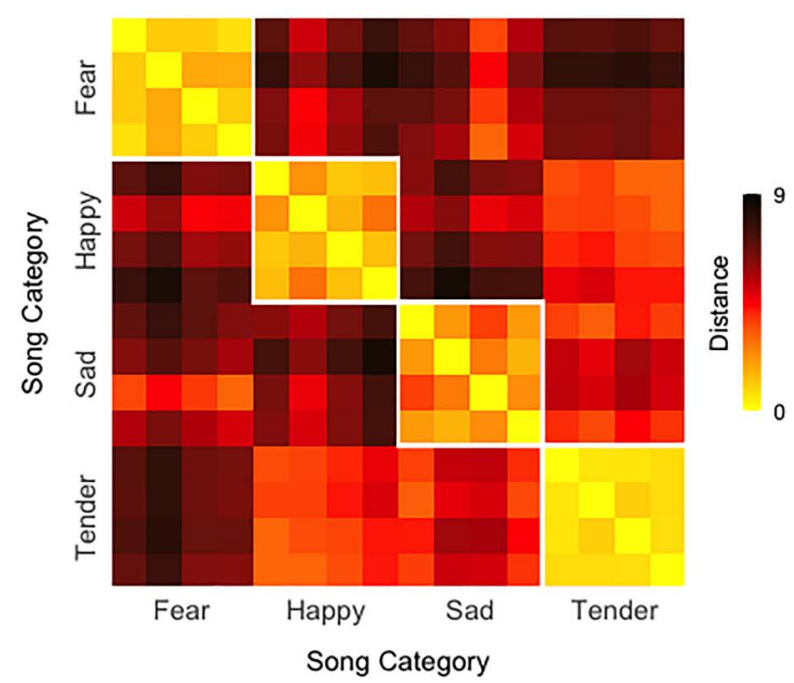

Figure 1. (a) Mean ratings for the intensity of each emotion for each musical excerpt. (b) Rating dissimilarity matrix (Euclidean distance) for each song pair.

leave-one-subject-out cross-validation tests the generalizability of the results across the sample of the subjects. A subjectwise GLM with regressors for each stimulus was fit to the data resulting in 16 beta weights (4 emotion categories $\times 4$ song per category) per voxel for each subject, and the MVPA was applied to the beta images. The beta weights were normalized to have zero mean and unit variance before application of MVPA. This analysis was performed using whole-brain data (with non-brain voxel masked out) and within a subset of the ROIs (amygdala, hippocampus, thalamus, anterior and posterior cingulate, SMA, precentral and postcentral gyri, precuneus, frontal pole, auditory cortex) where emotion classification has been successful in previous studies (Paquette et al. 2018; Saarimäki et al. 2015). In the whole-brain MVPA, an ANOVA feature selection was applied to the training set within each cross-validation where 5000 voxels with the highest $F$ score were selected. Classification accuracy was quantified by computing the proportion of correctly classified songs relative to the number of songs in each of the four categories (i.e., recall). To estimate the null distribution for the classification accuracies (naïve chance level 25\%), the following procedure was repeated 500 times: we 1) randomly shuffled emotion category labels; 2) ran the whole-brain MVPA with 102 leave-one-subject-out cross-validations, where the classifier was trained on the data with shuffled labels from $\mathrm{N}-1$ subject and tested on data with correct labels from the remaining subject; and 3) calculated the classification accuracies on each of the 500 iterations. If the true accuracy was larger than $95 \%$ of the accuracies obtained with the randomly shuffled labels, the true accuracy was considered significant with an alpha of 0.05 .

\section{Results}

\section{Behavioral Ratings}

The emotion ratings indicated that the musical excerpts reliably induced the target emotions (Fig. 1a), and most pieces elicited moderate feelings of liking (mean: 5.7, Supplementary Figure 1). The pieces were rated consistently across the subjects for fear, happiness, sadness, tenderness, and liking (mean inter-subject correlation: 0.68). Repeated-measure ANOVAs revealed significant main effects of song category on the mean ratings for each emotion $(P<0.001$; for all emotions, see Supplementary Materials for the full ANOVA results). Pair-wise comparisons of fear, sadness, happiness, and tenderness ratings across the scary, sad, happy, and tender songs showed that each song category was rated highest for the target emotion (i.e., the scary songs were rated higher for fear than the other songs etc., $P<0.001$ for all contrasts). Liking ratings were lower for the scary songs than for any other song category ( $P<0.05$ for all comparisons) and lower for the rock songs than for the happy songs $(P<0.01)$. We computed dissimilarity matrices (Euclidean distances) for each song pair to illustrate the similarity/dissimilarity of rating profiles within and across the emotion categories (Fig. 1b). The dissimilarity matrix indicates that the rating profiles were more similar within than across categories, confirming that the emotional experiences were similar for songs within a given category and distinct from the experience induced by the songs in the other categories.

\section{Brain Responses Evoked by Emotional Music}

We first modeled brain responses to each musical emotion dimension. Fear elicited subcortical activity bilaterally in the brainstem, thalamus, putamen, and pallidum. Activity was also observed in the amygdala and the insula (Fig. 2). There was extensive bilateral activity across the precentral gyrus and SMA extending into the postcentral gyrus. A cluster of activity was also found in the cerebellum. Frontal activity was found in the right inferior frontal gyrus and bilaterally in the frontal operculum and frontal pole. The cingulate gyrus was activated across both anterior and posterior portions. Occipital activity was observed in the precuneus. Finally, there was activity across the auditory cortical regions including Heschl's gyrus, planum temporale, planum polare, and suprerior temporal gurys. Happiness elicited right-lateralized activity in the pallidum and putamen. The insula was activated bilaterally. Parietal activity was observed in the SMA. Frontal activity was found in the right frontal pole. There were significant clusters of activity 

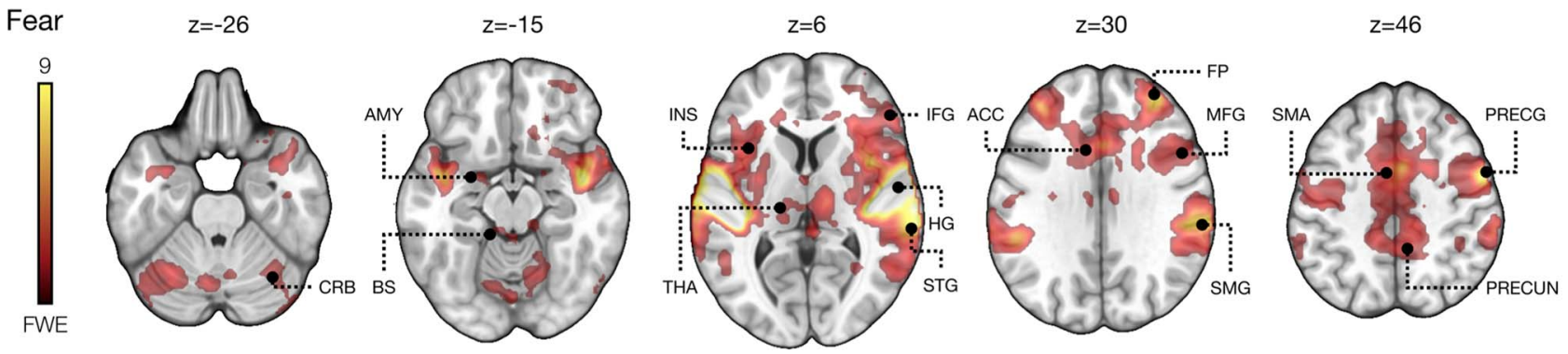

Happiness $\quad z=-6$

$\mathrm{z}=12$

$\mathrm{z}=6$

$z=30$

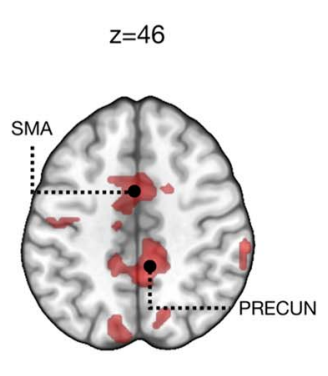

FWE
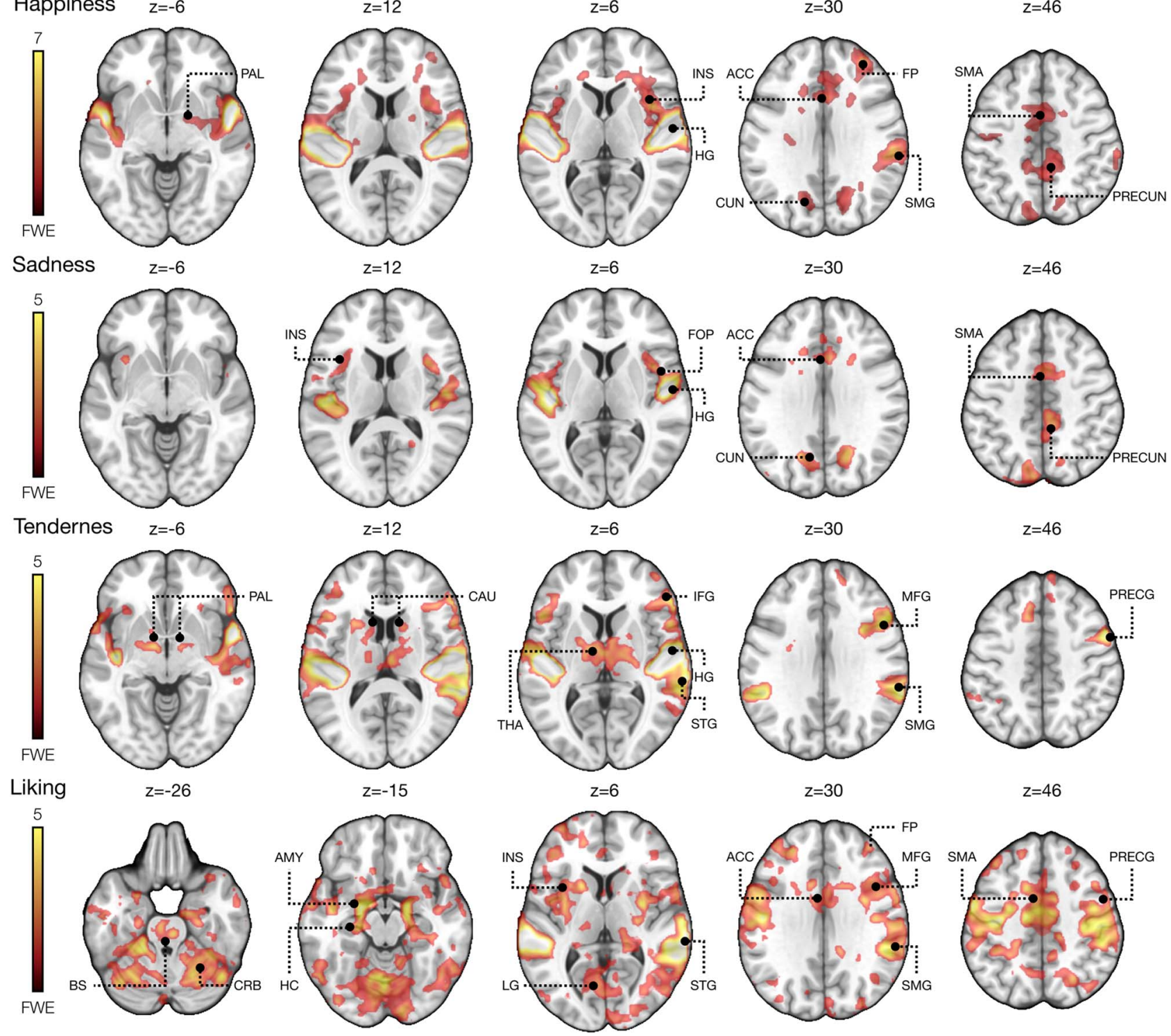

Figure 2. Brain regions responding to fear, happiness, sadness, tenderness and liking. The data are thresholded at $P<0.05$ and FWE corrected at the cluster level. $\mathrm{ACC}=$ anterior cingulate, $\mathrm{AMY}=$ amygdala, $\mathrm{CAU}=$ caudate, $\mathrm{CBR}=$ cerebellum, $\mathrm{CUN}=$ cuneus, $\mathrm{FOP}=$ frontal operculum, $\mathrm{FP}=$ frontal pole, $\mathrm{HC}=$ hippocampus, $\mathrm{HG}=$ Heschl's gyrus, $\mathrm{IFG}=$ inferior frontal gyrus, INS=insula, $L G=$ lingual gyrus, MFG=middle frontal gyrus, $P A L=$ pallidum, PRECG=precentral gyrus, PRE$\mathrm{CUN}=$ precuneus, $\mathrm{SMA}=$ supplementary motor area, $\mathrm{SMG}=$ supramarginal gyrus, $\mathrm{STG}=$ superior temporal gyrus, $\mathrm{THA}=$ thalamus. The color bar indicates the T value.

in the anterior and posterior divisions of the cingulate gyrus. Occipital activity was observed in the cuneus and precuneus. Finally, there was activity in Heschl's gyrus extending into planum temporale. Sadness activated the insula and frontal operculum, anterior cingulate gyrus, cuneus/precuneus, and Heschl's gyrus. Tenderness elicited subcortical activity in the thalamus, pallidum, and the caudate. Occipital activity was found in the right precentral gyrus. Frontal activity was observed 
(a) Cumulative maps of brain responses associated with music-induced emotions
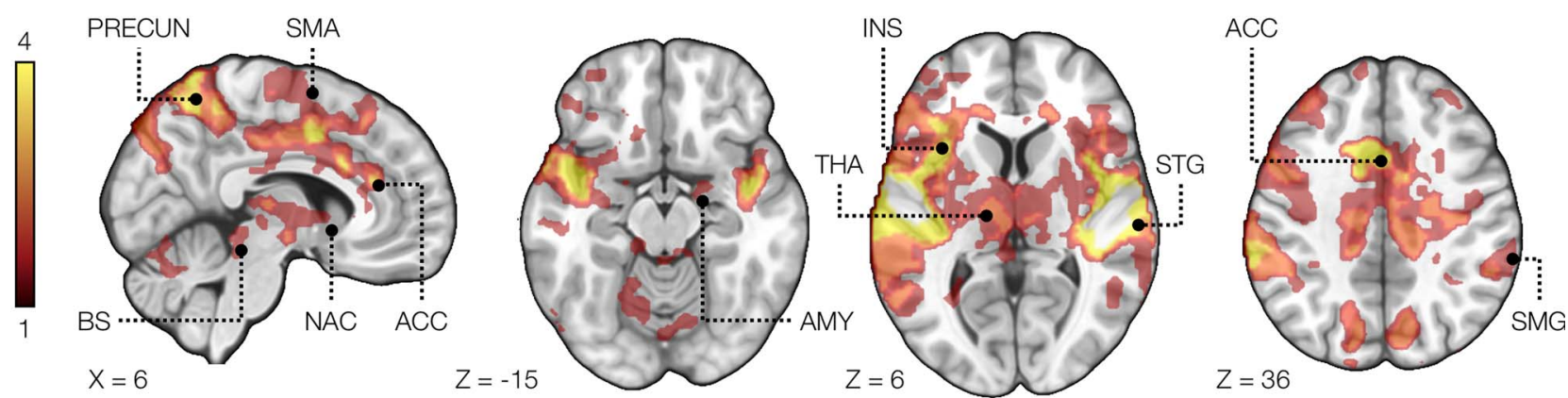

(b) Brain responses to emotion-inducing film clips
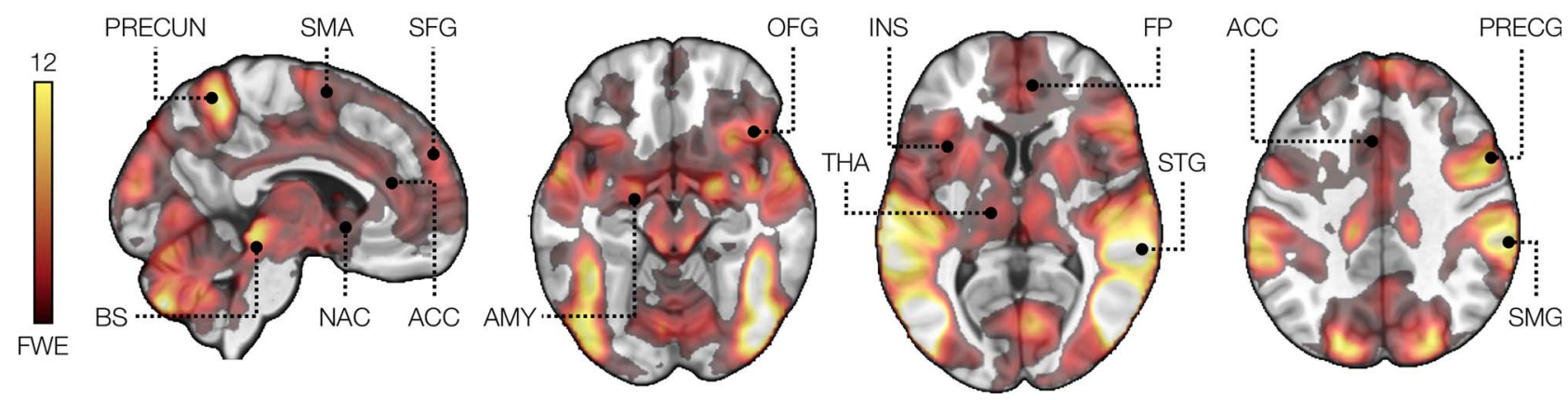

Figure 3. (a) Cumulative regional map of music-evoked activations. Voxel intensities show the number of musical categories (across fear, happiness, sadness, and tenderness) showing statistically significant $(P<0.05 \mathrm{FWE})$ activations at each voxel. $(b)$ Brain regions responding to emotional stimulation in the film experiment. The data are thresholded at $\mathrm{P}<0.05$ and FWE corrected at the cluster level $\mathrm{ACC}=$ anterior cingulate, $\mathrm{AMY}=\mathrm{amygdala}, \mathrm{FP}=$ frontal pole, $\mathrm{INS}=$ insula, $\mathrm{PRECG}=\mathrm{precentral}$ gyrus, PRECUN = precuneus, SMA = supplementary motor area, STG= superior temporal gyrus, THA=thalamus. The color bar indicates the $\mathrm{T}$ value.

in inferior, middle, and superior frontal gyri and the frontal pole. Significant clusters were also found bilaterally in the supramarginal gyrus. Finally, there was extensive activity in the auditory cortical regions encompassing Heschl's gyrus, planum temporale, planum polare, and superior temporal gyrus. Liking activated the brainstem, hippocampus and parahippocampal gyrus, amygdala, putamen, and thalamus. Extensive activity was observed across the somatosensory and motor regions in the precentral and postcentral gyri, SMA, and cerebellum. Frontal activity was observed bilaterally in the inferior, middle, and superior frontal gyri and frontal pole. Occipital activity was seen in the lingual gyrus. Significant clusters were also found bilaterally in the supramarginal gyrus and the superior temporal gyrus. To assess which regions were most consistently activated by different emotions, we first binarized the aforementioned statistically thresholded activation maps for each emotion (fear, happiness, sadness, tenderness) and generated a summed image of the maps. The resulting cumulative activation map (Fig. 3a) thus shows how many emotion categories activated each voxel at the a priori (FWE 0.05) statistical threshold.

\section{Brain Responses to Emotional Videos}

In the GLM analysis of the video experiment data, the emotion intensity regressor revealed activation in various regions associated with emotion such as the brainstem, thalamus, ventral striatum (nucleus accumbens, NAcc), amygdala, insula and the cingulate gyrus, and the orbitofrontal cortex (Fig. 3b). There was also extensive activation in the midline frontal regions and across somatomotor regions in the precentral gyrus, SMA, and cerebellum. Occipital activity was observed in the precuneus and in regions involved in visual processing. Temporal activity was observed in the auditory cortical regions.

\section{Regional Effects to Emotional Music}

To parse out the emotion specificity of the regional responses, we next performed ROI analyses for the music-evoked responses (Fig. 4). Only fear and liking significantly activated the amygdala, putamen, and ACC at the ROI level. Furthermore, significant hippocampal activation observed only for liking. Happiness activated the insula which was also activated by fear. Tenderness activated the thalamus and pallidum. Sadness did not reveal any significant effects in the analyzed ROIs, and no effects were observed in the caudate.

\section{Emotion Classification with MVPA}

For the whole-brain four-class MVPA, the mean accuracy was $49 \%$ and each of the four emotions were classified significantly above chance level (fear: $51 \%$, happiness: $45 \%$, sadness: $53 \%$, tenderness: $48 \%$, all $\mathrm{P}<0.001$ ). The 5000 most important voxels for the multi-class classification were predominantly located in the bilateral auditory cortices (Fig. 5a). Accordingly, the ROI-level MVPA revealed that classification accuracy for all four emotions was clearly above chance level for the auditory cortex (mean accuracy $48 \%$ ) and reached significance also in the precentral gyrus (mean accuracy 29\%) (Figs $5 b$ and 6). Although the overall classification accuracy significantly exceeded the chance level 

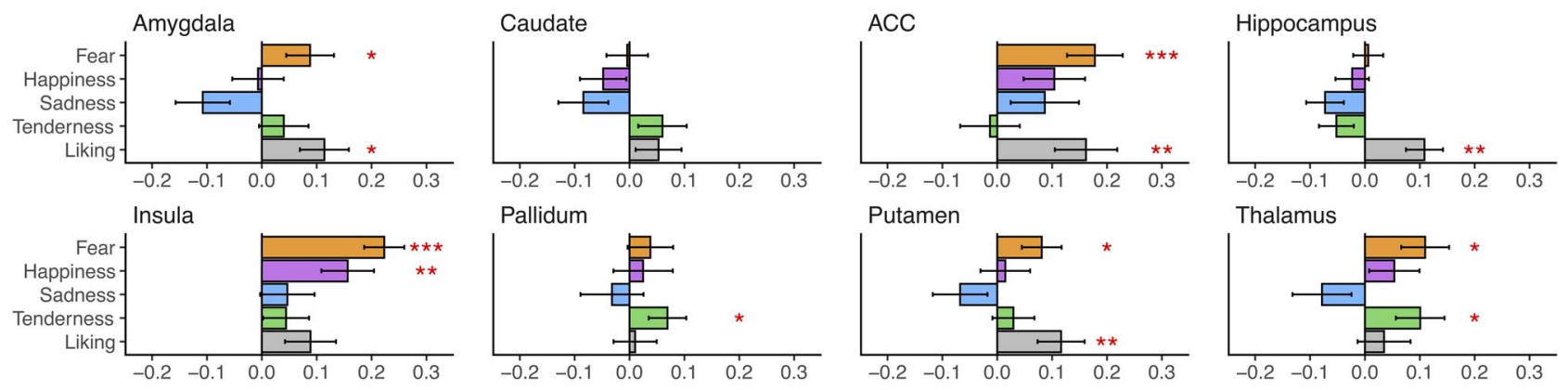

Figure 4. Mean beta weights (arbitrary scale) for each emotion regressor in each ROI. The error bars indicate standard error of mean and asterisks denote significant one-sample $t$ tests against zero. The regional results are plotted for visualization purposes only; statistical inference is based on the full-volume analysis. ${ }^{*}<<0.05$, $* * P<0.01, * * * P<0.001$

(a)

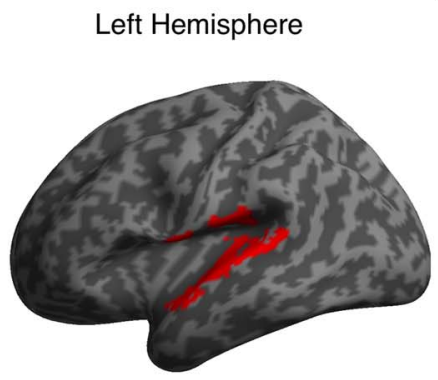

Right Hemisphere

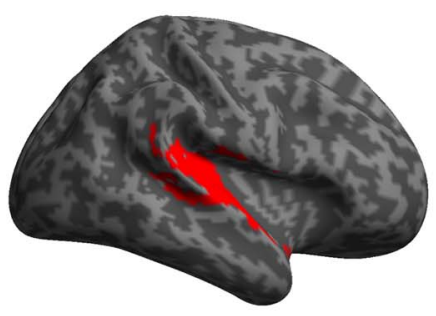

(b)
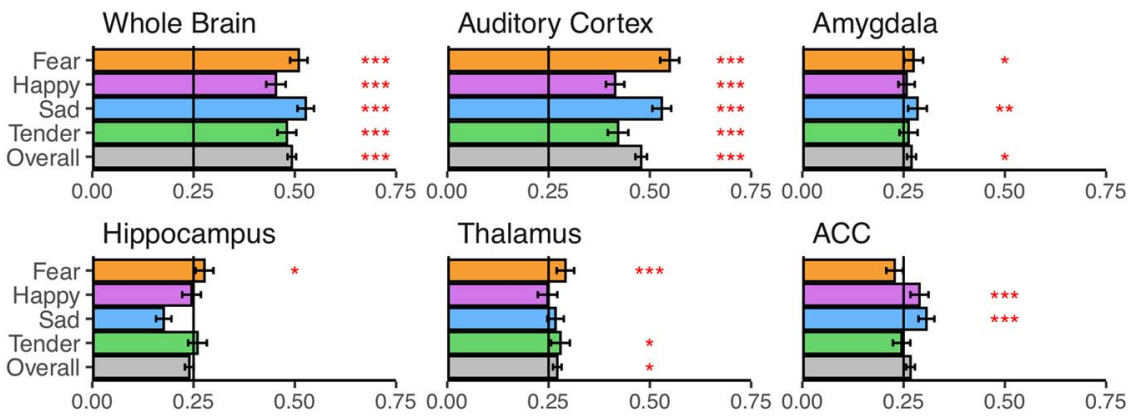

Posterior Cingulate

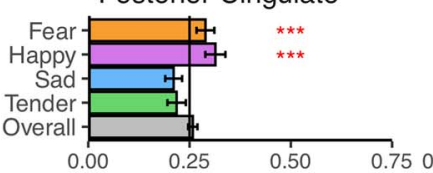

Precentral Gyrus

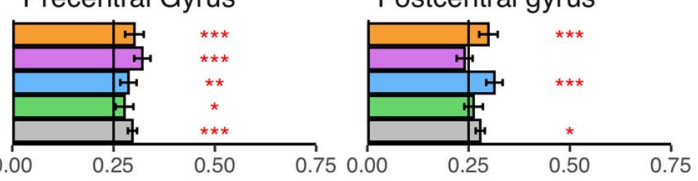

SMA

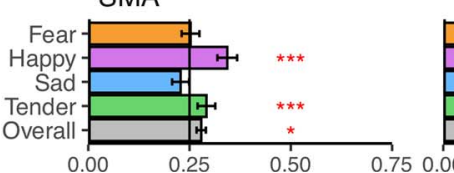

Precuneus

Frontal Pole
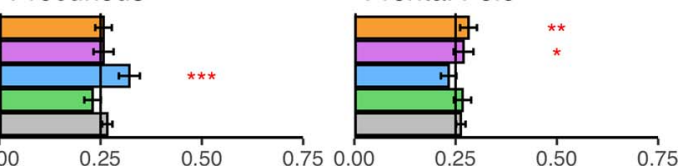

Figure 5. (a) The most important voxels for the between-subject classification of emotion categories in the whole-brain analysis. (b) Emotion-wise classification accuracies for the whole-brain and regional MVPA. ${ }^{*} P<0.05,{ }^{*} P<0.01,{ }^{*} * * P<0.001$.

for the amygdala, thalamus, precentral and postcentral gyri, SMA, and precuneus ROIS, the classification accuracies were dramatically lower than that of the auditory cortex. Furthermore, none of these ROIs significantly discriminated between all four emotions (Fig. 5b, Supplementary Figure 5). The ROI analyses were also performed separately for the left and right hemispheres, but these analyses revealed no substantial hemispheric differences and the classification accuracies tended to be lower than for the bilateral ROI analyses (see Supplementary Figure 5).

\section{Discussion}

Our main finding was that music-induced fear, happiness, sadness, and tenderness have discrete neural representations in the auditory and motor cortices. The GLM results showed that the music-induced emotions activated a common network comprising the auditory cortices, somatosensory/motor regions (SMA, precentral and postcentral gyri, cerebellum), cingulate cortex, insula, and precuneus, yet classification accuracies did not consistently exceed chance level in any region outside auditory and motor cortices. Moreover, music-induced emotions resulted in weak activation in limbic regions such as the ventral striatum, thalamus, and amygdala and cortical areas such as medial prefrontal and orbitofrontal cortices which are implicated in non-musical emotions (Lindquist et al. 2012) and were strongly activated by the emotional film clips. These findings cast doubt on the argument that different music-induced emotions engage the affective circuits in a discrete fashion (c.f. Saarimäki et al. 2015,2018 ) and instead suggest that these emotions involve a relatively unspecific activation outside the auditory and motor cortices.

\section{Auditory and Motor Cortical Representations of Music-Induced Basic Emotions}

Despite high statistical power and subjective reports of strong music-evoked emotion, we found only limited evidence that 

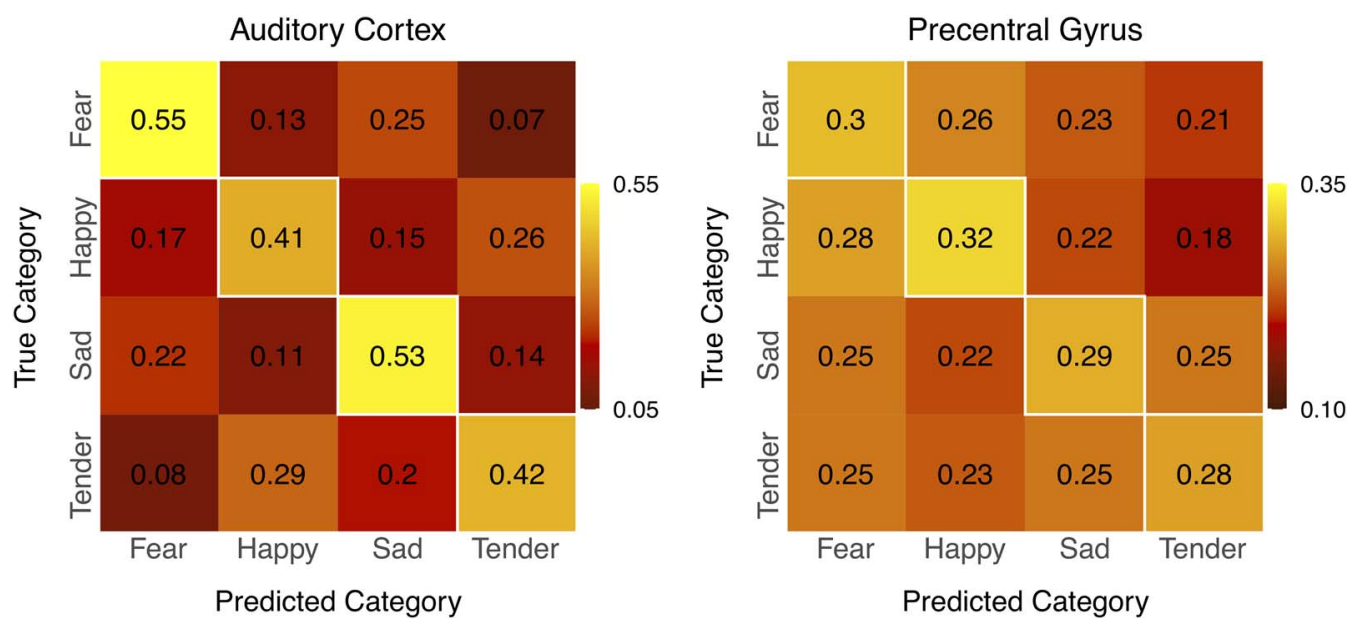

Figure 6. MVPA confusion matrices for the auditory cortex and precentral gyrus ROIs. The numbers indicate the proportion of true positives (diagonal) and false negatives within each emotion category.

music-induced emotions consistently engage the same core limbic circuits as emotions with survival value. The GLM analysis revealed extensive auditory cortical activation for all four emotions as well as liking. This accords with previous studies showing that the auditory cortices are more strongly activated by happy and sad than neutral music (Mitterschiffthaler et al. 2007) and that hemodynamic response in the auditory cortex tracks dynamic arousal ratings during music listening (Trost et al. 2012) and when listening to speech describing emotional episodes with neutral prosody (Nummenmaa et al. 2014). This effect likely reflects enhanced, general sensory gain for the emotionally salient stimuli. In the whole-brain MVPA, activity patterns in the auditory cortices also predicted with high accuracy whether the subjects were listening to a scary, happy, sad, or tender piece indicating that the BOLD signal in bilateral auditory cortices carries sufficient information for distinguishing categories of musical pieces conveying different discrete emotions. The classification accuracies we observed in the auditory cortex ROIs are comparable with those reported in prior pattern classification studies on musical (Paquette et al. 2018; Sachs et al. 2018) and non-musical emotions (Saarimäki et al. 2015). Tonal, rhythmic, and timbral differences between sad, happy, scary, and tender music (Laurier et al. 2009) presumably contribute to these emotion-specific auditory cortical activity patterns. These patterns were consistent across individuals, as evidenced by the successful leave-one-subjectout cross-validation. In the regional-level MVPA, the auditory cortex showed high classification accuracy for all emotions, whereas the accuracies in all other ROIS constituting central nodes of the emotion circuit were substantially lower or at chance level.

For the whole-brain and auditory cortex ROI, the classification accuracies for happiness and tenderness were slightly lower than for fear and happiness. The auditory cortex confusion matrix shows that these categories were somewhat more difficult to distinguish from one another than the other categories. Furthermore, the rating dissimilarity matrix suggests that the emotional experience was slightly more similar between happy and tender pieces (mean Euclidean distance: 3.82 ) relative to the other categories (mean Euclidean distance across other category pairs: 6.01). Thus, the similarity in emotional experience was reflected in similarity of the corresponding brain activity patterns in the auditory cortex. This probably reflects the fact that both categories included pleasant sounding pieces in major key that received high liking ratings.

Primary motor cortex (precentral gyrus) was the only ROI outside the auditory cortex where classification accuracy exceeded the chance level for all the tested emotions. The SMA and postcentral gyrus ROIs also showed above chance-level overall classification accuracy, although the accuracies for all individual emotions did not consistently reach significance in these ROIs. In the GLM, most emotions also activated regions involved in motor control and somatosensation such as the precentral and postcentral gyri, SMA, supramarginal gyrus, and cerebellum. This accords with data implicating somatomotor regions in emotion perception and the subjective experience of emotions (Adolphs et al. 2000; Pourtois et al. 2004; Nummenmaa et al. 2012) and studies indicating that pleasurable music engages motor regions such as the SMA and cerebellum more strongly than emotionally less evocative music (Blood and Zatorre 2001; Pereira et al. 2011). Music often induces spontaneous rhythmic movements in the listener which is evident already in young children (Zentner and Eerola 2010) and even in some non-human animals (Hattori and Tomonaga 2020). Thus, the sensorimotor activity observed in the current study might reflect this perception-movement coupling even in the absence of overt movement (cf. Zatorre et al. 2007). Furthermore, different music-induced emotions prime distinct types of movements (Burger et al. 2013) which could explain the covariation of activity in the motor regions and emotion ratings in the GLM and the emotion-specific multivoxel activity patterns in the primary motor cortex.

Two prior MVPA-fMRI studies (Paquette et al. 2018; Sachs et al. 2018) have found evidence for differential activity patterns for fear, happiness, and sadness in the auditory cortex, and importantly, neither of these could reveal the emotion-specific activity pattern within the limbic regions. Arguably, these results might stem from use of short $(<2 \mathrm{~s})$ sound bursts, from the "Musical Emotional Bursts" stimulus set (Paquette et al. 2013) as stimuli which probably did not evoke strong subjective emotions in the subjects (although neither of these studies report the intensity of the felt emotions in response to the stimuli but only accuracy of behavioral classification to the a priori emotional 
categories). However, despite 1) using naturalistic music as stimuli rated relatively high on each target emotion and 2) having high statistical power with 102 subjects, the present categorical approach provided no evidence of emotion-specific activation outside the auditory and motor cortices. This is in clear contrast with pattern recognition studies in other domains which have found that emotion categories are represented in a distributed fashion across cortical and subcortical regions (Kragel and LaBar 2016; Nummenmaa and Saarimäki 2017). A metaanalysis of neuroimaging studies also indicates that different basic emotion categories can be predicted across studies from activity patterns that span several intrinsic functional networks (Wager et al. 2015), suggesting that emotions elicited by nonmusical stimuli arise from interactions between systems that also support processes that are not exclusively affective such as memory, attention, and action. Music-induced emotions might differ from more prototypical basic emotions by relying more heavily on sensory or aesthetic processing and consequently on more spatially localized auditory cortical representations.

\section{Music-Induced Emotions and Core Emotion Circuits}

The video experiment for mapping networks governing nonmusical emotions revealed extensive and strong activation in regions involved in emotional processing including the amygdala, thalamus, NAcc, ACC, and insula and midline frontal regions. Although the MVPA did not reveal consistent emotionspecific patterns outside the auditory and motor cortices, the GLM for the music-induced emotions did reveal activation in many (e.g., insula, ACC, amygdala) although not all (e.g., midline frontal regions) components of these emotion circuits.

Both the music-induced emotions (except for tenderness) and those induced by the film clips activated the ACC and insula. Previous studies indicate that ACC activity is positively associated with the intensity of pleasurable music-induced chills (Blood and Zatorre 2001) and responds to happy music (Mitterschiffthaler et al. 2007). The insula is implicated in the processing of interoceptive feedback (Craig 2002) and is likely involved in generating the somatic component of emotional experience (Nummenmaa et al. 2018). ACC and insula activation has consistently been coupled with autonomic arousal suggesting that these regions contribute to the physiological changes (e.g., in heart rate, blood pressure, respiration, and muscle tone) that typically accompany emotions (Beissner et al. 2013). Accordingly, ACC and insula activation was particularly extensive for the high arousal emotions fear and happiness. Activation of the precuneus was also observed for most of the music-induced emotions and for the video experiment in line with previous studies, showing that this region is activated by different task involving introspection (Cavanna and Trimble 2006).

The GLM also confirmed the contribution of the amygdala in music-induced fear in accordance with human lesion studies (Gosselin et al. 2005) and some prior fMRI studies (Aubé et al. 2015; Koelsch et al. 2013; however, see Bogert et al. 2016), implicating the amygdala in music-induced fear. However, liking also activated this region in line with data showing that the amygdala responds to a range of non-aversive but salient stimuli and is not exclusively involved in fear-related functions but contributes to the detection of emotional significance more generally (Sander et al. 2003; Pessoa 2010). Accordingly, amygdala activation has previously been reported in response to both pleasant and unpleasant music compared to neutral music (Ball et al. 2007) and joyful music compared to scary music (Koelsch et al. 2006; for a meta-analysis, see Koelsch 2014).

The liking ratings also predicted activity in the hippocampus. Prior studies have associated activation in the hippocampal formation with music-induced positive emotions like tenderness (Trost et al. 2012) and joy (Mueller et al. 2011), pleasantness of musical chords (Cheung et al. 2019), and the perception of happiness in music (Bogert et al. 2016) but also with the processing of unpleasant dissonant versions of pleasant consonant music (Koelsch et al. 2006). A cluster comprised of the hippocampus and adjacent structures was observed in a meta-analysis on the neural correlates of music-induced emotions and was proposed to mediate the stress reduction by music and music-induced positive, attachment-related emotions (Koelsch 2014). Another study found that liked vs. disliked music activated many of the same regions whose activity correlated with the liking ratings in the current study including the caudate, thalamus, parahippocampal gyrus, anterior cingulate, superior temporal gyrus, precuneus, and the cerebellum (Brattico et al. 2016). Another structure implicated in pleasure, liking, and positive emotions in across studies is the NAcc (Koelsch 2014). However, in contrast to previous studies (Blood and Zatorre 2001; Salimpoor et al. 2013), we did not observe an association between liking and activity in the nucleus accumbens in the ventral striatum. This discrepancy might be partly due to the fact that, unlike the studies providing strongest evidence for Nacc contribution in music-induced pleasure, we did not target individuals particularly prone to experience music-induced frisson (Salimpoor et al. 2013; MartínezMolina et al. 2016) and used unfamiliar music as stimuli (cf. Pereira et al. 2011). Furthermore, some studies reporting NAcc activity have contrasted liked music with highly unpleasant, dissonant music (Menon and Levitin 2005; Mueller et al. 2015). In the current study, all musical stimuli received relatively high liking ratings which may have precluded the detection NAcc activity.

It is possible that the weaker limbic and paralimbic responses to music versus emotional videos simply reflect the weaker emotional potency of the musical excerpts. Alternatively, it is possible that videos contain more overlapping features driving the limbic circuits.

In sum, the GLM results indicate that music-induced emotions may engage central nodes of the core emotion circuits (cf. Koelsch, 2004) although the activation of these circuits was substantially less extensive for the music stimuli than for the videos. It is possible that the weaker limbic and paralimbic responses to music versus emotional videos simply reflect the weaker emotional potency of the musical excerpts. Alternatively, it is possible that videos contain more temporally overlapping dynamic features (e.g., facial expressions, social interaction) driving the limbic circuits (Lahnakoski et al. 2012). Irrespective of the ultimate answer to this question, activity patterns in these regions did not consistently differentiate specific music-induced emotions in the MVPA, suggesting that activity in these circuits does not contain neural signatures of distinct music-induced emotions. Prior studies indicate that limbic and paralimbic regions are activated by a range of positive and negative emotions (Wager et al. 2015) perhaps because these regions govern some elementary ingredients of emotion such as encoding of valence, arousal, or motivational relevance shared by all emotions. The current study suggests that, together with the emotion-specific representations in the auditory and motor cortices, these circuits give rise to subjective music-induced affective states that listeners interpret as categorically distinct basic emotions such as happiness and sadness. 


\section{Limitations}

We used unfamiliar instrumental music as stimuli to minimize the contribution of autobiographical memories and lyrics to the emotional responses. This attempt to maximize experimental control may have resulted in weaker emotional reactions than, for example, with familiar music with lyrics. Indeed, previous studies indicate that familiar music elicits stronger emotional reactions (Peretz et al. 1998) and engages the brain's reward circuits more strongly than unfamiliar music (Pereira et al. 2011). Our self-reports are consistent with strong affective engagement (Fig. 1), yet it is also possible that these results may be inflated due to demand characteristics. Finally, it is possible that the dimensional valence arousal or music-specific categorical models would better describe how music-induced emotions are organized which would arguably make our analysis approach suboptimal for detecting affect-related brain responses.

\section{Conclusion}

We conclude that music-induced emotions are represented as discrete patterns of activity in the auditory and motor cortices. These emotions consistently engage a network of auditory cortical areas (Heschl's gyrus, planum polare, planum termporale) and regions supporting motor control, somatosensation, and interoceptive processing (pre- and post-central gyri, SMA, cerebellum, ACC, insula, precuneus). Despite high statistical power, we did not find evidence for music-induced basic emotions which rely strongly on the subcortical limbic system or medial frontal regions that govern basic emotions induced by biologically salient stimuli. We propose that even though listeners often interpret their subjective responses to music in terms of basic emotion categories, these feelings may only partially rely on the same neural machinery as prototypical basic emotion with adaptive significance.

\section{Supplementary Material}

Supplementary material can be found at Cerebral Cortex online.

\section{Funding}

Academy of Finland (\#294897 and \#332225 to L.N.); European Research Council Starting Grant (\#313000 to L.N.); Sigrid Juselius foundation grant to LN.

\section{References}

Adolphs R, Damasio H, Tranel D, Cooper G, Damasio AR. 2000. A role for somatosensory cortices in the visual recognition of emotion as revealed by three-dimensional lesion mapping. J Neurosci. 20:2683-2690.

Aubé W, Angulo-Perkins A, Peretz I, Concha L, Armony JL. 2015. Fear across the senses: brain responses to music, vocalizations and facial expressions. Soc Cogn Affect Neurosci. 10:399-407.

Ball T, Rahm B, Eickhoff SB, Schulze-Bonhage A, Speck O, Mutschler I. 2007. Response properties of human amygdala subregions: evidence based on functional MRI combined with probabilistic anatomical maps. PLoS One. 2:e307.

Beissner F, Meissner K, Bär K-J, Napadow V. 2013. The autonomic brain: an activation likelihood estimation meta-analysis for central processing of autonomic function. J Neurosci. 33:10503-10511.

Blood AJ, Zatorre RJ. 2001. Intensely pleasurable responses to music correlate with activity in brain regions implicated in reward and emotion. Proc Natl Acad Sci. 98:11818-11823.

Bogert B, Numminen-Kontti T, Gold B, Sams M, Numminen J, Burunat I, Lampinen J, Brattico E. 2016. Hidden sources of joy, fear, and sadness: explicit versus implicit neural processing of musical emotions. Neuropsychologia. 89:393-402.

Brattico E, Alluri V, Bogert B, Jacobsen T, Vartiainen N, Nieminen S, Tervaniemi M. 2011. A functional MRI study of happy and sad emotions in music with and without lyrics. Front Psychol. 2:308.

Brattico E, Bogert B, Alluri V, Tervaniemi M, Eerola T, Jacobsen T. 2016. It's sad but I like it: the neural dissociation between musical emotions and liking in experts and laypersons. Front Hum Neurosci. 9:676.

Burger B, Saarikallio S, Luck G, Thompson MR, Toiviainen P. 2013. Relationships between perceived emotions in music and music-induced movement. Music Percept Interdiscip J. 30:517-533.

Cavanna AE, Trimble MR. 2006. The precuneus: a review of its functional anatomy and behavioural correlates. Brain. 129:564-583.

Cheung VKM, Harrison PMC, Meyer L, Pearce MT, Haynes JD, Koelsch S. 2019. Uncertainty and surprise jointly predict musical pleasure and amygdala, hippocampus, and auditory cortex activity. Curr Biol. 29:4084-4092.e4.

Craig AD. 2002. How do you feel? Interoception: the sense of the physiological condition of the body. Nat Rev Neurosci. 3:655-666.

Çukur T, Nishimoto S, Huth AG, Gallant JL. 2013. Attention during natural vision warps semantic representation across the human brain. Nat Neurosci. 16:763-770.

De La Vega A, Chang LJ, Banich MT, Wager TD, Yarkoni T. 2016. Large-scale meta-analysis of human medial frontal cortex reveals tripartite functional organization. J Neurosci. 36:6553-6562.

Eerola T, Vuoskoski J. 2010. A comparison of the discrete and dimensional models of emotion in music. Psychol Music. 39:18-49.

Ekman P. 1992. An argument for basic emotions. Cognit Emot. 6:169-200.

Escoffier N, Zhong J, Schirmer A, Qiu A. 2013. Emotional expressions in voice and music: same code, same effect? Hum Brain Mapp. 34:1796-1810.

Esteban O, Markiewicz CJ, Blair RW, Moodie CA, Isik AI, Erramuzpe A, Kent JD, Goncalves M, DuPre E, Snyder M et al. 2019. fMRIPrep: a robust preprocessing pipeline for functional MRI. Nat Methods. 16:111.

Ethofer T, Van De Ville D, Scherer K, Vuilleumier P. 2009. Decoding of emotional information in voice-sensitive cortices. Curr Biol. 19:1028-1033.

Fonov V, Evans A, McKinstry R, Almli C, Collins D. 2009. Unbiased nonlinear average age-appropriate brain templates from birth to adulthood. NeuroImage, Organization for Human Brain Mapping 2009 Annual Meeting. 47:S102.

Fritz T, Jentschke S, Gosselin N, Sammler D, Peretz I, Turner R, Friederici AD, Koelsch S. 2009. Universal recognition of three basic emotions in music. Curr Biol. 19:573-576.

Frühholz S, Trost W, Kotz SA. 2016. The sound of emotionstowards a unifying neural network perspective of affective sound processing. Neurosci Biobehav Rev. 68:96-110. 
Gosselin N, Peretz I, Noulhiane M, Hasboun D, Beckett C, Baulac M, Samson S. 2005. Impaired recognition of scary music following unilateral temporal lobe excision. Brain. 128: 628-640.

Hanke M, Halchenko YO, Sederberg PB, Hanson SJ, Haxby JV, Pollmann S. 2009. PyMVPA: a python toolbox for multivariate pattern analysis of fMRI data. Neuroinformatics. 7: 37-53.

Hattori Y, Tomonaga M. 2020. Rhythmic swaying induced by sound in chimpanzees (Pan troglodytes). Proc Natl Acad Sci. 117:936-942.

Higgins KM. 2012. Biology and Culture in Musical Emotions. Emot Rev. 4:273-282.

Heikkilä TT, Laine O, Savela J, Nummenmaa L. 2020. Onni: An online experiment platform for research (Version v1.0). Zenodo. http://doi.org/10.5281/zenodo.4305573.

Juslin PN. 2013. What does music express? Basic emotions and beyond. Front Psychol. 4:596.

Juslin PN, Laukka P. 2003. Communication of emotions in vocal expression and music performance: different channels, same code? Psychol Bull. 129:770-814.

Juslin PN, Vastfjall D. 2008. Emotional responses to music: the need to consider underlying mechanisms. Behav Brain Sci. 31:559-575.

Karjalainen T, Karlsson HK, Lahnakoski JM, Glerean E, Nuutila P, Jääskeläinen IP, Hari R, Sams M, Nummenmaa L. 2017. Dissociable roles of cerebral $\mu$-opioid and type 2 dopamine receptors in vicarious pain: a combined PET-fMRI study. Cereb Cortex. 27:1-10.

Karjalainen T, Seppälä K, Glerean E, Karlsson HK, Lahnakoski JM, Nuutila P, Jääskeläinen IP, Hari R, Sams M, Nummenmaa L. 2019. Opioidergic regulation of emotional arousal: a combined PET-fMRI study. Cereb Cortex. 29:4006-4016.

Kassam KS, Markey AR, Cherkassky VL, Loewenstein G, Just MA. 2013. Identifying emotions on the basis of neural activation. PLoS One. 8:e66032.

Kim J, Shinkareva SV, Wedell DH. 2017. Representations of modality-general valence for videos and music derived from fMRI data. Neuroimage. 148:42-54.

Kober H, Barrett LF, Joseph J, Bliss-Moreau E, Lindquist K, Wager TD. 2008. Functional grouping and cortical-subcortical interactions in emotion: a meta-analysis of neuroimaging studies. Neuroimage. 42:998-1031.

Koelsch S. 2014. Brain correlates of music-evoked emotions. Nat Rev Neurosci. 15:170-180.

Koelsch S, Fritz T, Cramon Y, Müller K, Friederici AD. 2006. Investigating emotion with music: an fMRI study. Hum Brain Mapp. 27:239-250.

Koelsch S, Skouras S, Fritz T, Herrera P, Bonhage C, Küssner MB, Jacobs AM. 2013. The roles of superficial amygdala and auditory cortex in music-evoked fear and joy. Neuroimage. 81:49-60.

Konečni VJ. 2008. Does music induce emotion? A theoretical and methodological analysis. Psychol Aesthet Creat Arts. 2:115-129.

Kragel PA, LaBar KS. 2015. Multivariate neural biomarkers of emotional states are categorically distinct. Soc Cogn Affect Neurosci. 10:1437-1448.

Kragel PA, LaBar KS. 2016. Decoding the nature of emotion in the brain. Trends Cogn Sci. 20:444-455.

Lahnakoski JM, Glerean E, Salmi J, Jääskeläinen IP, Sams M, Hari R, Nummenmaa L. 2012. Naturalistic fMRI mapping reveals superior temporal sulcus as the hub for the distributed brain network for social perception. Front Hum Neurosci. 6:233.
Laurier C, Lartillot O, Eerola T, Toiviainen P. 2009. Exploring relationships between audio features and emotion in music. In: Louhivuori J, Eerola T, Saarikallio S, Himberg T, Eerola PS, editors. Proceedings of the 7th Triennial Conference of European Society for the Cognitive Sciences of Music. Jyväskylä: University of Jyväskylä Press. pp. 260-264.

Lindquist KA, Wager TD, Kober H, Bliss-Moreau E, Barrett LF. 2012. The brain basis of emotion: a meta-analytic review. Behav Brain Sci. 35:121-143.

Martínez-Molina N, Mas-Herrero E, Rodríguez-Fornells A, Zatorre RJ, Marco-Pallarés J. 2016. Neural correlates of specific musical anhedonia. Proc Natl Acad Sci. 113:E7337-E7345.

McDermott MT, Schultz AF, Undurraga EA, Godoy RA. 2016. Indifference to dissonance in native Amazonians reveals cultural variation in music perception. Nature. 535:547-550.

Menon V, Levitin DJ. 2005. The rewards of music listening: response and physiological connectivity of the mesolimbic system. Neuroimage. 28:175-184.

Mitterschiffthaler MT, Fu CHY, Dalton JA, Andrew CM, Williams SCR. 2007. A functional MRI study of happy and sad affective states induced by classical music. Hum Brain Mapp. 28:1150-1162.

Mueller K, Fritz T, Mildner T, Richter M, Schulze K, Lepsien J, Schroeter ML, Möller HE. 2015. Investigating the dynamics of the brain response to music: a central role of the ventral striatum/nucleus accumbens. Neuroimage. 116:68-79.

Mueller K, Mildner T, Fritz T, Lepsien J, Schwarzbauer C, Schroeter ML, Möller HE. 2011. Investigating brain response to music: a comparison of different fMRI acquisition schemes. Neuroimage. 54:337-343.

Nummenmaa L, Glerean E, Viinikainen M, Jääskeläinen IP, Hari R, Sams M. 2012. Emotions promote social interaction by synchronizing brain activity across individuals. Proc Natl Acad Sci. 109:9599-9604.

Nummenmaa L, Hari R, Hietanen JK, Glerean E. 2018. Maps of subjective feelings. Proc Natl Acad Sci. 115:9198-9203.

Nummenmaa L, Saarimäki H. 2017. Emotions as discrete patterns of systemic activity. Neurosci Lett. 69:3-8.

Nummenmaa L, Saarimäki H, Glerean E, Gotsopoulos A, Jääskeläinen IP, Hari R, Sams M. 2014. Emotional speech synchronizes brains across listeners and engages large-scale dynamic brain networks. Neuroimage. 102:498-509.

Paquette S, Peretz I, Belin P. 2013. The "musical emotional bursts": a validated set of musical affect bursts to investigate auditory affective processing|psychology. Front Psychol. 4:509.

Paquette S, Takerkart S, Saget S, Peretz I, Belin P. 2018. Crossclassification of musical and vocal emotions in the auditory cortex: cross-classification of musical and vocal emotions. Ann N Y Acad Sci. 1423:329-337.

Peelen MV, Atkinson AP, Vuilleumier P. 2010. Supramodal representations of perceived emotions in the human brain. $J$ Neurosci. 30:10127-10134.

Pereira CS, Teixeira J, Figueiredo P, Xavier J, Castro SL, Brattico E. 2011. Music and emotions in the brain: familiarity matters. PLoS One. 6:e27241.

Peretz I. 2010. Towards a neurobiology of musical emotions. In: Handbook of music and emotion: theory, research, applications. Oxford, England: Oxford University Press, pp. 99-126.

Peretz I, Gaudreau D, Bonnel A-M. 1998. Exposure effects on music preference and recognition. Mem Cognit. 26:884-902.

Pessoa L. 2010. Emotion and cognition and the amygdala: from "what is it?" to "what's to be done?". Neuropsychologia. 48:3416-3429. 
Pourtois G, Sander D, Andres M, Grandjean D, Reveret L, Olivier E, Vuilleumier P. 2004. Dissociable roles of the human somatosensory and superior temporal cortices for processing social face signals. Eur J Neurosci. 20:3507-3515.

Pruim RH, Mennes M, van Rooij D, Llera A, Buitelaar JK, Beckmann CF. 2015. ICA-AROMA: a robust ICA-based strategy for removing motion artifacts from fMRI data. Neuroimage. 112:267-277.

Saarimäki H, Ejtehadian LF, Glerean E, Jääskeläinen IP, Vuilleumier P, Sams M, Nummenmaa L. 2018. Distributed affective space represents multiple emotion categories across the human brain. Soc Cogn Affect Neurosci. 13:471-482.

Saarimäki H, Gotsopoulos A, Jääskeläinen IP, Lampinen J, Vuilleumier P, Hari R, Sams M, Nummenmaa L. 2015. Discrete neural signatures of basic emotions. Cereb Cortex. 26:2563-2573.

Sachs ME, Habibi A, Damasio A, Kaplan JT. 2018. Decoding the neural signatures of emotions expressed through sound. Neuroimage. 174:1-10.

Salimpoor VN, van den BI, Kovacevic N, McIntosh AR, Dagher $A$, Zatorre RJ. 2013. Interactions between the nucleus accumbens and auditory cortices predict music reward value. Science. 340:216-219.

Sander D, Grafman J, Zalla T. 2003. The human amygdala: an evolved system for relevance detection. Rev Neurosci. 14:303-316.

Scherer K, Zentner M. 2008. Music evoked emotions are different-more often aesthetic than utilitarian. Behav Brain Sci. 31:595-596.
Sitaram R, Lee S, Ruiz S, Rana M, Veit R, Birbaumer N. 2011. Realtime support vector classification and feedback of multiple emotional brain states. NeuroImage, Multivariate Decoding and Brain Reading. 56:753-765.

Sonkusare S, Breakspear M, Guo C. 2019. Naturalistic stimuli in neuroscience: critically acclaimed. Trends Cogn Sci. 23:699-714.

Trost W, Ethofer T, Zentner M, Vuilleumier P. 2012. Mapping aesthetic musical emotions in the brain. Cereb Cortex. 22:2769-2783.

Volynets S, Smirnov D, Saarimäki H, Nummenmaa L. 2020. Statistical pattern recognition reveals shared neural signatures for displaying and recognizing specific facial expressions. Soc Cogn Affect Neurosci. 15:803-813.

Vuoskoski JK, Eerola T. 2012. Can sad music really make you sad? Indirect measures of affective states induced by music and autobiographical memories. Psychol Aesthet Creat Arts. 6:204.

Wager TD, Kang J, Johnson TD, Nichols TE, Satpute AB, Barrett LF. 2015. A Bayesian model of category-specific emotional brain responses. PLoS Comput Biol. 11:e1004066.

Zatorre RJ, Chen JL, Penhune VB. 2007. When the brain plays music: auditory-motor interactions in music perception and production. Nat Rev Neurosci. 8:547-558.

Zentner M, Eerola T. 2010. Rhythmic engagement with music in infancy. Proc Natl Acad Sci. 107:5768-5773.

Zentner M, Grandjean D, Scherer KR. 2008. Emotions evoked by the sound of music: characterization, classification, and measurement. Emotion. 8:494-521. 